\title{
A VISUAL CONTENT ANALYSIS OF SCHOOL IMAGES AND SIGNS: THEIR EFFECT ON HIGH SCHOOL STUDENTS
}

\author{
Sofia Tsagdi, \& Konstantinos Theologou \\ National and Technical University of Athens (Greece)
}

\begin{abstract}
Visual methods are often marginalized in educational research and have not been employed to collect information about cultural identities of the school and its effect on the students. The aim of this presentation is to examine visual methods for understanding the visual culture of schools and how these images are perceived and processed by high school students in Greece. It reports on a participative research project in four secondary schools in Greece from distinctively different cultural and economic backgrounds. The strategy of research applied in this study is grounded theory and the qualitative methods of research are: structured interviews (4 interviews done and transcribed during one month), scaled questionnaires were distributed ( 80 done during one month and transcribed) and photography (800 photos done during one month and described) and repeated visits in schools. There were at least 80 students involved at the project during one month. Moreover this presentation draws on content analysis as a systematic, rigorous approach to analyzing documents obtained or generated in the course of research. Finally the presentation will conclude that these approaches provide a comprehensive view of how visual images are produced and interpreted, and of what their potential social consequences may be. The use of visual methods is not without challenges however. Securing ethics approval and school participation along with problems with camera retrieval and protecting participant agency were some difficulties encountered in the current study. For those wishing to pursue less conventional research methodologies in educational settings, this presentation will also highlight potential benefits and struggles.
\end{abstract}

Keywords: School culture, visual images, multimodality, participatory social research, hidden curriculum.

\section{Introduction}

Being a language teacher nearly every text that I looked at and implemented in my teaching, used two modes of communication: (a) language as writing and (b) image. However, when I was to teach and involve my students in activities I realized that I merely focused on language as the only medium that represented fully the meanings I wanted to encode and communicate. Images were used as stimulators of a pre task activity at their most. They were in my mind simply somebody else's job.

The same applied to all the images put around school. Me, as well as most educators, considered them either as purely decorative or a as just a visual aid to attract attention to the text. In our mind it was the text that forged the values, beliefs, customs that hold the social group together. Drawing predominantly on the work of Kress (1997) and others (Kress \& Van Leeuwen, 1996) in social semiotics and multimodality, I thought it was time to unsettle this common sense notion and take things a step further. I began this process by conceptualizing the school environments as semiotic spaces in which human beings, who are the agents of their own meaning making produce multimodal texts - visual, written, spoken, performative, sonic, and gestural. In the act of making meaning and expressing their ideas students/teachers, produce multiple signs in textual and non-textual forms across semiotic modes, drawing on different representational resources in order to succeed in that domain. Furthermore, students shape their understanding of the world not only through the texts we present to them. Rather, they form their ideas through these implicit powerful forces, hidden in images and visuals. Moreover, we must note that the design/presentation of such texts/images is constrained by the genres, languages, and discursive practices that are valued within the broader sociocultural and political context of education and the nation-state. I felt that our duty as educators was to collect these images and analyze their meaning in order to better understand the social cultural forces of a school, since as it is argued that they shaped the organization's outcomes (Rutter et al. 1979). 


\section{Methodology}

Our sample consisted of 10 schools chosen from areas, with a wide variety of economic and social background. Economic zone ranking of the Ministry of Economics was used in order to indicate the extent to which a school draws its students from low or high socio-economic communities. Moreover, rankings from the Ministry of Education, that rank schools according to their performance in university entrance exams were also taken into consideration, while selecting the schools. Student population was ethnically diverse comprising mainly of Greek students, but with a significant number of students from Albanian and Middle East origins. The staff was only from Greek origin however. Two hundred students and 50 teachers participated in the research. In total, 65\% of the participants self-defined as European the $20 \%$ as of Balkan origin and the rest as of Middle East origin.

The procedure followed was: First the researcher, applied for the consent of the Ministry of Education to ensure access to the schools and their collaboration to research. This process took about 3 months to be completed. Afterwards, the researcher contacted the principal of the school to arrange a convenient date to visit the school and take pictures and video of the images in and outside school premises. Also, she emailed the school the parent consent form that should be distributed to students in order to have their parents' permission to answer questionnaires and participate in the research. Then, in the arranged date she took photos of the school, in one day after school hours, in order not to interrupt school life but also so as not to have issues of shooting students while trying to capture an image. This made the research logistically possible while offering a discrete moment of school life to analyze. Afterwards, the images were examined and ten most prominent regarding their place in school as well as their frequency of message, were developed. The images were classified in the following categories: Classification of visuals

The visuals were first classified in categories relating to who was responsible for producing and placing them. Teacher-Students or Other. Then they were classified in one of the 20 categories: related to courses, racial, insults, sexual insults, general insults, sexual humor, general humor, dominant gender (male female) romanticism, politics, drugs, religion, morals, names, sports, personal problems, art, music, sexuality, philosophical remarks, and miscellaneous. The researcher then returned to school, usually after two days, to distribute questionnaires to both teachers and students (usually 20 students participated). After the questionnaires were collected the researcher displayed in print the ten collected images (the most prominent in school) and initiated an informal discussion on students' feelings about these images or about any other images the students wanted to talk about and the researcher had not put forward. The researcher kept notes of their responses.

\section{Results}

From the visual collected $81 \%$ was placed in the school by teachers particularly Technology, Biology and IT teachers. The messages teachers displayed, were either mainly related to courses $20 \%$ or to general issues. The most common being Environmental issues with smaller percentages of Art. The remaining percent of schools visuals involved graffiti and visual messages carved or drawn by students on desks, chairs or outside school usually during lessons or after school hours. Most of these visuals and messages, concerned sexuality (18.9\%) and sports. The proportion of student produced visuals concerned sexuality in addition to the categories of sexual request, sexual humor, sexual insults and remarks was $41 \%$. The second highest proportion was reference to sport teams (15\%). Of the 20 categories of graffiti, sexuality, sports-music and politics were those found most frequently in student produced messages. (18.4\% and $15.4 \%$, respectively). What seems to be particularly interesting is that schools that could be classified as of a high socio-economic status had political messages put by students' supporting left wing political parties that are traditionally associated with the working class. Another interesting finding is that of the "non legitimate" and offensive messages $70 \%$ were produced by male students while female students appear to produce messages relating to philosophy, art and courses. Also reported in the study is that visual with romantic content were found more frequently in women's desks chairs or restrooms than in men's. This anonymous school testimony bears witness to the importance of emotional relationships for women (Mellen, 1998). All the categories of visuals were present at some level in the male's visual messages, whereas racial insults, references to drugs, and references to sex were absent from the women's. Female's visual messages were more conservative and conventional than male's; morality and religion represented $11.6 \%$ of the content of the female's visual messages but only $2.1 \%$ of the male's. We also need to note that male's visual messages were prevalent and dominant outside the schools building while female's were limited inside the premises. A final point that needs to be pointed out is that although the messages students of all backgrounds produced could almost be equally fitted in the above classifications, in school of low economic background the percent of racial, ethnic and racist remarks was 
very high. Also, as it can easily be seen from the sample of the images shown. In the schools of low ranking and socioeconomic background there is a distinct absence of visuals and images "formally" displayed, while this gap is "filled" by students" own messages on school walls and equipment. Finally in no school discussion among staff and students, as of what should be on the school space was ever conducted. The most that was asked of the students was to draw a theme that would later be displayed by staff around the school.

\section{Discussion}

Drawing on the results of the survey we could argue that schools of low socioeconomic background were exposed to very few visual messages and where visual were present they only portray images that showed school values and rules. In Schools of high socioeconomic rank, however, there are more and more images of school activities in schools particularly since digital photography has made it so easy to capture events and activities for displays. Many of these images reinforced positive messages about pupils and about the opportunities the school provides for excitement, enjoyment thereby playing a significant role in reinforcing/promoting desirable norms and establishing ideals. However, this use neglected much of their potential contribution to educational processes and to the development of school as educational institutions. There were groups of teachers, as well as students that wanted to portray images that showed their pride in their school and images that portray school values that otherwise could be lost. Many teachers mostly focused on the displays and pictures that showed school performance in certain subjects such as technological projects and participation in European programs i.e youth parliament, Erasmus etc. We need to point out though an important finding. In the low income schools students felt freer to place messages around the school. However, at the same time they felt unsatisfied with the appearance of the school that seemed to them as neglected.

In the case of the high income schools most students however focused on images that were linked to aesthetically pleasant results and on messages, and again on images mainly produced by students. Then, there were some mostly older students that focused on images linked to school management and relationship with their peers. This last point highlights the contribution that images can make to informed discussion of the perspectives of those whose critical voice goes unheard.

The students thoughts and feelings interestingly matched the percentages of visuals. Meaning that where, for instance, sports were more prominent the interests of students and the discussion did evolve around this topic. Accordingly, in schools were visuals of cultural issues were present students seemed more aware, sensitized and willing to talk about issues in such areas. They felt mostly satisfied with the appearance of their school, though not free to put visuals or interact with the school space. We must note that every month they were asked to clean their desks of any messages and the schools was maintained and painted annually. Another finding, was that in high income schools the community, especially the parents association were allowed and given space to put messages inside the school, usually of informative nature about forthcoming activities.

We need to say that while the perspectives of teacher and even parents are often well represented in qualitative school based educational research the perspectives of students are often marginalized if not ignored entirely (Elliot 1991). Since pupils have the least amount of power in school communities (only through the 15 elected board) and the least say in terms of their education, authorities make it even more complicated and difficult for students to engage in research. Yet in my opinion it is these marginalized pupils opinions that are of most value in stimulating institutional change. Because they are the most strongly subject to the taken for granted and unquestioned by those who are more powerful. Interviews and questionnaires although difficult to obtain were not adequate methods to unravel students' perspectives. Student's also reported that our research offered the students and staff an opportunity to view their school from their and other's perspectives. Our research was thought by students, as something "out of the ordinary" an interruption of the routine. Some of the students also valued the fact that their opinion not only was asked but also that it was treated as equal to those of their teachers. They valued that they were treated as equals in the process, although in practice their different status afforded them minor involvement in the educational processes.

It was interesting to find that images such as (01) signified different things and provoked different to students and staff. Image (02) in most students of the school provoked feelings of happiness and joy while for teachers it seemed as self-evident, unimportant signifying emptiness and loss. However, it was also found that certain images provoked a shared perspective between teacher and students mostly images that had to do with litter and environmental issues.

However, we must point out that, there were many hurdles that needed to be overcome for our research to be feasible. Obtaining consent to enter the school was time consuming and involved a lot of bureaucracy. Another major challenge this project faced was securing schools' participation. Even with 
the ministry's permission most schools were reluctant to participate and posed several hurdles on the process. Particularly most school principal wanted to check the photos taken and usually insisted on deleting photos that contained messages/signs that could diminished authority or could portray the school as neglected by the principal. Faced with a method not commonly used and not anticipated the principal expressed considerable anxiety and skepticism. The teachers' and principal's reactions reflect wider anxieties about camera-use at school. A number of high profile media cases involving students taking photos on mobile phones and distributing them via the internet have heightened fears about this issue (Netsafe 2005). Some of the schools enforce strict rules around camera phones and punish students for inappropriate use. When cameras were routinely perceived as problematic their research use was perceived too perilous and as undermining school authority. Anxiety about cameras appears to invoke a 'double standard' with photographs viewed potentially more exposing and dangerous than written text. Emmison and Smith (2000) reveal this point in their discussion of authors' and editors' reluctance to include photographs in published works:

What is ironic, we suggest, is that whilst photographs are often deemed to be unacceptable by authors and editors, textually explicit descriptions of morally suspect materials are considered less so. Such a 'double standard' tells us quite a lot about the relationship of our society to the image as opposed to the text. Whilst texts are associated with reason and higher mental faculties, images are seen as subversive, dangerous and visceral. (Emmison and Smith 2000, 14)

This unease related to the school being identifiable even with the use of a pseudonym. The research was only allowed to proceed on my guarantee measures would be taken to prevent the school's identification.

School-based research can also be hindered, by congested timetabling also hampers accommodating 'an extra' like research, when academic pursuits take precedence. In addition, the need to negotiate alterations to ethical approval prolonged fieldwork. Although time-intensive, acting on teachers' suggestions was a means signaling their concerns were valued. Key to participation in schools was the ministry's consent and supportive teachers who considered the research beneficial to students and helped quell senior management's anxieties. Securing school participation was subsequently a challenge as the research was constituted as 'too risky' by principals anxious to avoid unwanted publicity.

During our research issues of gender, sexuality emerged since, visual images/messages regarding gender and sexuality were the second most commonly produced by students. However, researching issues of sex representation and gender issues in schools is challenging because it asks questions about an issue which is socially constituted as 'private', 'embarrassing', "non relevant to educational goals" "political" and subsequently 'problematic'. As a result, the researcher was not allowed by the present class teacher to discuss such issues with the students even though students did point out several times such issues, drawing from the pictures shown to them. In some occasions, even such visual were not permitted to be shown at all to students, though they were collected in the school and had a prominent presence in the school space.

To conclude, it was of major interest to see that visual around the school do shape and express the values of the school and the local community. To take things further, the researcher shape the belief that using visual methods can provoked students into thinking further of what they took for granted. Also, Images of a school can act as a contact zone, where teachers and students of different ages of ethnic groups can come together, if not on equal terms, nevertheless in a place were communication is possible. The images and texts can foreground different perspectives which provide different questions and alternative to the dominant accounts of schooling. None of this however is possible if visuals are not "seen" and explored in the educational process.

\section{References}

Allen, L. 2008. Young people's agency in sexuality research using visual methods. Journal of Youth Studies, 11(6): 565-77. [Taylor \& Francis Online], [Web of Science ®]][Google Scholar]

Casey, C. 2001. "Ethics committees, institutions and organisations; subjectivity, consent and risk". In Research ethics in Aotearoa New Zealand: Concepts, practice, critique, Edited by: Tolich, Martin. 127-40. Auckland: Pearson Education New Zealand. [Google Scholar]

Emmison, M. and Smith, P. 2000. Researching the visual: Images, objects, contexts and interactions in social and cultural inquiry, London: Sage.

Felstead, A., Jewson, N. and Walters, S. 2004. "Images, interviews and interpretations: Making connections in visual research". In Studies in qualitative methodology: Seeing is believing? Approaches to visual research, Edited by: Pole, Christopher. 105-21. Oxford: Elsevier. 
Knowles, C. and Sweetman, P. 2004. Picturing the social landscape: Visual methods and the sociological imagination, London: Routledge.)

Latham, A. 2004. Researching and writing everyday accounts of the city. In Picturing the social landscape: Visual methods and the sociological imagination, ed. Caroline Knowles and Paul Sweetman, 117-31. London: Routledge.

Netsafe. 2005. The text generation mobile phones and New Zealand youth: A report of results from the internet safety group's survey of teenage mobile phone use

Pink, S. 2001. Doing visual ethnography. London: Sage

Ramazanoglu, C. and Holland, J. 2002. Feminist methodology: Challenges and choices, London: Sage.

Weedon, C. 1987. Feminist practice and post-structuralist theory, 2nd ed., Oxford: Blackwell. 